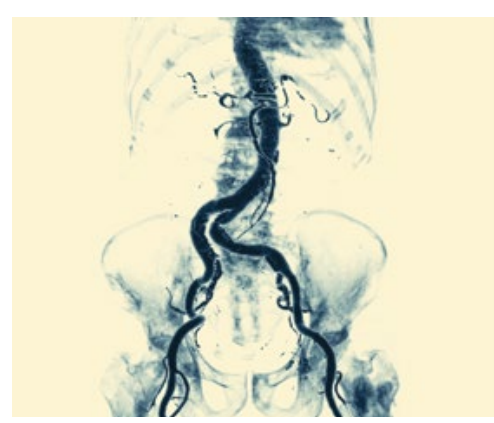

CPD

Mayo Theivendran, Jason Chuen

\section{Background}

Screening and diagnostic surveillance of latent conditions have a profound impact on public healthcare expenditure and clinical outcomes. Abdominal aortic aneurysm (AAA) remains one of the hallmark pathologies in vascular surgery and an area of intense research interest.

\section{Objectives}

This article is the second of two that will outline current areas of controversy and research in AAA disease in order to support a more detailed understanding of issues in managing patients with this condition, and inform the development of Australasian clinical guidelines and health policy.

\section{Discussion}

Screening and surveillance of AAA should be evidence-based and follow clinical guidelines; however, advances in treatment technology and epidemiological data have influenced results. Goals of care and cost-effectiveness should play central parts in screening and surveillance strategies.

\title{
Updates on AAA screening and surveillance
}

UNLIKE MANY OTHER medical conditions, an abdominal aortic aneurysm (AAA) is generally asymptomatic and detected incidentally through clinical examination, imaging investigations for other conditions, or targeted screening studies. Unfortunately, intervention at the time of symptomatic presentation (namely AAA rupture) carries high mortality and morbidity. Therefore, the primary focus of treatment is early identification in asymptomatic patients and treatment at clinically recommended thresholds.

The aim of this article is to review current information on population and targeted screening for AAA, and the role of surveillance imaging in the lead-up to and after surgical repair in greater depth than previous summaries. It is assumed that readers are familiar with the basic concepts described in previous papers on aortic and non-aortic aneurysms. ${ }^{1,2}$

\section{Population screening for abdominal aortic aneurysm}

There are no official AAA screening programs in Australia, despite population screening programs in the UK and Sweden, where there is an $80 \%$ uptake, ${ }^{3}$ and numerous other European countries. Australian Medicare rules prohibit reimbursement for population screening studies; however, targeted screening of individual patients, on the basis of age, smoking history and family history of AAA, can be requested by any medical practitioner and is funded through the Medicare Benefits Schedule in Australia as part of clinically justified investigations.
On the basis of evidence showing substantial benefit, the US Preventive Services Task Force (USPSTF) recommends one-time ultrasonography screening for men aged 65-75 years who have ever smoked. ${ }^{4}$ Selective screening for men in the same age group but with no smoking history still offers moderate public health benefit. However, no clear benefit exists for screening of women, even those with a smoking history. Longterm assessment of the Multicentre Aneurysm Screening Study (MASS) randomised trial ${ }^{5}$ demonstrated an approximate halving of mortality rates in men aged 65-74 years with screening. There were also health economic benefits, with a reported $£ 7600$ gain over a 10 -year period. Outcomes from the UK NHS ${ }^{6}$ and Swedish ${ }^{7}$ Nationwide AAA screening programs support these findings.

Interestingly, the Western Australian screening trial $^{8}$ is the only randomised trial so far to report no benefit from population screening. It is postulated that the lack of benefit is due to a high rate of incidental imaging or high levels of detection and treatment of AAA through routine clinical care in the Western Australian population, along with the challenges of delivery to a geographically remote population.

Most recently, in the Danish Viborg Vascular (VIVA) screening trial, a randomised controlled study of patients with AAA, peripheral arterial disease and hypertension, screening of 50,156 participants did not show any reduction in AAA-related deaths but did show an absolute risk reduction in all-cause mortality of 0.006 (admittedly, with wide 
confidence intervals [CI] of 0.001, 0.011) with one preventable death for each 169 (89-1811) men invited to participate. ${ }^{9}$

The justification for screening programs has been further revisited by the publication of a 2017 meta-analysis ${ }^{10,11}$ of all reported trials, which concluded that screening reduces AAA-related deaths (odds ratio [OR] 0.66, 95\% CI: 0.47, $0.93, P<0.02)$. A reduction in overall mortality in the invited men was also shown (hazard ratio [HR] 0.98, 95\% CI: 0.96, 0.99, $P=0.003)$. Identification of men with smaller aneurysms not indicated for intervention $(<5 \mathrm{~cm})$ allows for cardiovascular best medical management, improving overall health and optimisation for future aortic intervention. These studies also show that men who do not accept the offer to be screened have a higher mortality rate for AAA-related and unrelated diseases, highlighting the value of healthcare engagement for these patients.

\section{Screening for related aneurysms}

There are no accepted recommendations regarding screening for thoracic aortic aneurysm (TAA) in patients with AAA; however, additional family history of AAA, hypertension, obesity, African-American ethnicity and a large AAA on presentation have been suggested as factors to trigger screening for TAA. Traditionally, familial screening has been recommended for primary relatives over the age of 65 years, but recent evidence suggests that this should also be extended to younger relatives in whom there is clinical evidence of a collagen, elastin or connective tissue disorder such as Marfan or Loeys-Dietz syndromes. There are data suggesting that $12.6 \%$ of patients treated for an AAA continue to develop a metachronous TAA, ${ }^{12}$ while $20-25 \%$ of patients with TAA have an associated AAA.

We suggest that all patients with AAA should be screened for TAA at the time of diagnosis, with plain X-ray in two planes or non-contrast computed tomography (CT). We also suggest that patients with a primary presentation of a large AAA should undergo at least echocardiography to exclude aortic root dilatation and bicuspid aortic valve or ascending arch aneurysm, as well as ultrasonography for popliteal artery aneurysm (PAA). Conversely, patients with newly diagnosed with TAA may also benefit from AAA and PAA screening.

\section{Post-detection surveillance regimen}

While aortic aneurysm surveillance is generally recommended on the basis of maximal aortic diameter, it is recognised that this is merely a crude surrogate marker for rupture risk (Figure 1). Current research explores materials modelling, ${ }^{13}$ computational fluid dynamics and finite element analysis in order to predict how aneurysms will grow and the potential location and timing of rupture. ${ }^{14}$ Biological markers are also being investigated to determine whether they are viable measures of aneurysm activity for the sake of clinical management. ${ }^{15}$

At this time, maximal aortic diameter remains the primary surveillance tool; however, significant nuance exists in the determination of this measurement. For example, in a tortuous aorta a transverse or axial diameter may overstimate the size of the aneurysm. Most specialists will use a maximal outer-to-outer wall diameter perpendicular to the line of flow on CT as the true reference diameter for an aneurysm.

Even this diameter may not be reflective. For example, in a saccular aortic aneurysm, it is debatable whether the diameter of the saccular component or the entire vessel represents the clinically relevant predictor of rupture risk. In general, surgeons will have a lower threshold for treatment of saccular aneurysms.

In general, suggested surveillance intervals have been based on rupture risk and expected growth rates, estimated from the size of the aneurysm. The UK Small Aneurysm Trial (UKSAT) ${ }^{16}$ observed expansion rates as follows:

- $1.9 \mathrm{~mm}$ per year for AAA $2.8-3.9 \mathrm{~cm}$

- $2.7 \mathrm{~mm}$ per year for AAA $4.0-4.5 \mathrm{~cm}$

- $3.5 \mathrm{~mm}$ per year for AAA $4.6-8.5 \mathrm{~cm}$

The 2010 European Society for Vascular Surgery (ESVS) guidelines ${ }^{17}$ were based on modelling data from the UKSAT data. ${ }^{18}$ Recently published US Society for Vascular Surgery (SVS) guidelines for AAA surveillance ${ }^{19}$ incorporating data from the RESCAN collaborators ${ }^{20}$ included cost-effectiveness factors in their recommendations; however, these factors may not be applicable to other jurisdictions or cohorts. No guidelines have been published or endorsed by the Australian and New Zealand Society for Vascular Surgery (ANZSVS) to date. It remains to be seen whether the new surveillance intervals suggested by the SVS will gain traction among vascular surgeons (Table 1).

\section{Postoperative surveillance}

Postoperative surveillance is required after open repair and endovascular repair for synchronous or metachronous aneurysmal degeneration of other arterial beds, and appreciation of complications and failure of repair. Postoperative surveillance for open and endovascular repair vary because of the nature of complications in these populations.

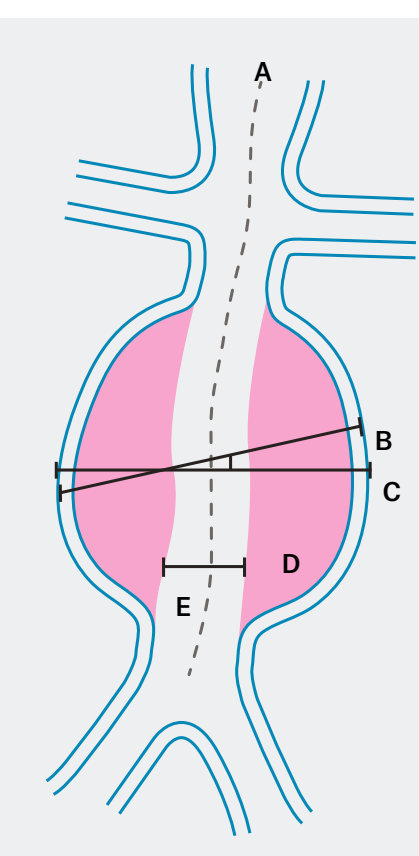

A. Centreline

B. False transverse diameter

C. True perpendicular diameter

D. Mural thrombus

E. Lumenal diameter

Figure 1. AAA measurement 


\section{Table 1. Suggested AAA surveillance intervals from SVS and ESVS}

\begin{tabular}{ll}
\hline $\begin{array}{l}2017 \text { SVS guidelines } \\
\text { based on RESCAN }\end{array}$ & $\begin{array}{l}2010 \text { ESVS guidelines } \\
\text { based on UKSAT modelling }\end{array}$ \\
\hline $2.5-3.0 \mathrm{~cm}: 10$ years & $3.0-3.9 \mathrm{~cm}: 24$ months \\
$3.0-3.9 \mathrm{~cm}: 3$ years & $4.0-4.5 \mathrm{~cm}: 12$ months \\
$4.0-4.9 \mathrm{~cm}: 12$ months & $4.5-5.0 \mathrm{~cm}: 6$ months \\
$5.0-5.4 \mathrm{~cm}: 6$ months & $>5.0 \mathrm{~cm}: 3$ months \\
\hline
\end{tabular}

ESVS, European Society for Vascular Surgery; SVS, Society for Vascular Surgery; UKSAT, UK Small Aneurysm Trial

There are no evidence-based guidelines for surveillance after open repair. Imaging is commonly performed six weeks after repair, for early evaluation of the operative site and graft, using ultrasonography or CT angiography (CTA), depending on body habitus. A follow up at 3-5 years is often performed for assessment of thoracoabdominal or aortoiliac aneurysmal progression, anastomotic false aneurysm or polyester graft degeneration, ${ }^{21}$ and then intermittently after that at the treating doctor's discretion. Delayed complications after open repair, such as synthetic graft infection, aorto-enteric fistula or anastomotic haemorrhage, are rare but can be disastrous. ${ }^{22}$

\section{Surveillance after endovascular aneurysm repair}

The integrity of endovascular aneurysm repair (EVAR) can be compromised by continued aneurysmal expansion, endoleak, migration of stents and component separation. Long-term surveillance consumes resources and may lead to unnecessary re-intervention. With a growing number of patients with EVAR, surveillance alone can consume the imaging resources of a high-volume centre. In this situation, it is desirable to survey patients who are at high risk for complications.

Graft failure after EVAR is not usually due to a single cause. Multiple factors contribute to failure in achieving long-term aneurysmal exclusion. The factors ${ }^{23}$ can broadly be categorised into:

- aneurysm anatomy (short neck, angulation, thrombus in neck, tortuous vessels, disease progression)
- device factors (integrity issues, poor design)

- physician factors (suboptimal device selection, implantation technique). With progressive evolution in EVAR experience, techniques and designs, lower long-term failure rates from EVAR are expected.

The early experience of EVAR mandated routine six-monthly CTA; however, although it is a sensitive, reproducible tool, there are issues with contrast nephropathy, cumulative lifetime radiation exposure and resource availability. Duplex ultrasonography (DUS) is a comparable tool ${ }^{24}$ but has limitations of operator variation and is difficult to coordinate across centres.

Current strategies have tried to minimise the use of repeated CTA on the basis that early freedom from endoleak predicts freedom from long-term aneurysm-related mortality at five years, ${ }^{25}$ and that early CTA indicators of highrisk can be used to guide the strictness of future surveillance. Similarly, early 30-day DUS negative for endoleak or limb stenosis appears predictive of a low re-intervention rate of $2 \%$ at three years. ${ }^{26}$

Stratification of patients into low risk and high risk in the early perioperative period can be used to select the population that requires strict follow-up with imaging. High-risk factors include unfavourable aneurysm anatomy, recognised suboptimal surgical issues, emergency or ruptured AAA repairs, and early-phase imaging evidence of endoleak or failure of sac shrinkage by $5 \mathrm{~mm}$ in 12 months.

Low-risk patients can be surveyed without the need for frequent CT beyond the first 12 months. The risk of further aneurysmal degeneration around the three-year mark deserves imaging in both low-risk and high-risk groups.

Most vascular surgeons will perform CTA at least once in the year after EVAR, interspersed with DUS and plain $\mathrm{X}$-ray. Indicators of possible endoleak are failed residual sac shrinkage on ultrasonography, or evidence of graft or component migration on X-ray or CT. Endoleaks can be detected on DUS, and sensitivity can be assisted with contrast enhancement, ${ }^{27}$ but confirmation usually requires delayed-phase CTA or selective angiography.

Surveillance of patients who are too fragile to undergo intervention for secondary repair or re-intervention should be reconsidered on an individual basis, with an emphasis on the patient's quality of life and minimal psychological distress. Essentially, cessation of surveillance should be considered for patients who will not be candidates for open repair or endovascular revision surgery.

\section{Conclusions}

Targeted population screening has been welcomed in some countries as there is evidence for a reduction in AAA-related deaths and improvement in cardiovascular outcomes in patients on surveillance, particularly men. We have previously outlined surveillance guidelines in our prior publications to assist surveillance once an AAA has been detected. These recommendations take into account the growth rate of the AAA and rupture risk at a given size.

The risk of graft failure and disease progression necessitates ongoing surveillance following EVAR. Tailored surveillance programs are used to minimise the resources allocated to ongoing surveillance. However, it is accepted that surveillance is paramount for long-lasting success with EVAR (Figure 2).

AAA disease continues to provide fertile ground for ongoing surgical research, and future developments will range from better surgical technique and medical devices, improved detection and counselling, the promise of pharmacotherapy, and 
risk assessment. Ultimately, screening programs, surgical repair and long-term surveillance should keep in mind the ultimate goal of preserving longevity and quality of life. Decisions should be grounded in realistic assessments of benefit and avoid futile or wasteful surgery or imaging.

\section{Screening}

- Opportunistic screening for incidental AAA during other investigations (eg CT/ultrasonography/MRI of the abdomen)

- Consider single-episode ultrasonography screening for AAA in:

- male patients aged $>65$ years with history of smoking

- patients aged $>65$ years with primary relative history of AAA

- adult patients with personal or primary relative history of Marfan, Loeys-Dietz or similar syndromes

- patients with known popliteal or thoracic aortic aneurysm

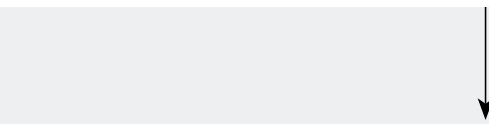

\section{Post-detection}

- Optimise cardiovascular and medical risk factors

- Refer to vascular surgeon for counselling, surveillance management, treatment planning and complex aneurysm decision making

- Current discrepancy in surveillance intervals

- suggest continuing with ESVS guidelines for now

- Screen for synchronous TAA or PAA, suggest familial screening

- Expedite surgical review if:

- unexplained abdominal pain/tenderness

- rapid expansion (generally defined as $>1 \mathrm{~cm} /$ year)

- diameter close to treatment threshold $(5.0 \mathrm{~cm}$ in women, $5.5 \mathrm{~cm}$ in men)

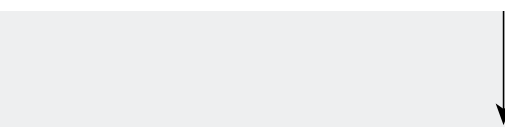

\section{Treatment}

- As determined by the vascular surgeon

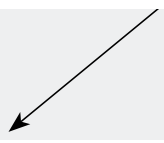

Post-treatment surveillance

- Open AAA repair

- Consider re-imaging at 3-5 years

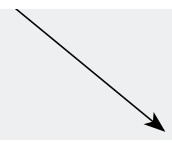

Post-treatment surveillance

- EVAR

- Lifelong surveillance, usually managed by vascular surgeon at 6-12 month intervals

Figure 2. Sample flowchart

AAA, abdominal aortic aneurysm; CT, computed tomography; ESVS, European Society for Vascular Surgery; EVAR, endovascular aneurysm repair; PAA, popliteal artery aneurysm; TAA, thoracic aortic aneurysm

\section{Authors}

Mayo Theivendran MBBS, FRACS (Vascular), FRACS (General), Vascular, Endovascular and Transplant Surgeon, North Shore Private, Sydney, St Vincent's Private, Sydney, the Northern Beaches Hospital, Sydney, NSW, and Department of Vascular Surgery, Austin Health, Melbourne Vic. mayotheivendran@ mac.com

Jason Chuen MBBS, FRACS (Vasc), MPH, PGDipSurg Anat, Director of Vascular Surgery, Department of Vascular Surgery, Austin Health, Vic; Department of Surgery, Faculty of Medicine, Dentistry and Human Sciences, The University of Melbourne, Vic

Competing interests: None.

Provenance and peer review: Commissioned, externally peer reviewed.

\section{References}

1. Robinson D, Mees B, Verhagen HJM, Chuen J. Aortic aneurysms - Screening, surveillance and referral. Aust Fam Physician 2013;42(6):364-69.

2. Mees B, Robinson D, Verhagen HJM, Chuen J. Non-aortic aneurysms - Natural history and recommendations for referral and treatment. Aust Fam Physician 2013;42(6):370-74.

3. Ross NP. Uptake of AAA screening. A cohort study. Eur J of Vasc Surg 2013;45(6):610-15.

4. LeFevre ML, U.S. Preventive Services Task Force. Screening for abdominal aortic aneurysm: U.S. Preventive Services Task Force recommendation statement. Ann Intern Med 2014;161(4):281-90. doi: 10.7326/M14-1204.

5. Thompson SG, Ashton HA, Gao L, Buxton MJ Scott RAP, Multicentre Aneurysm Screening Study (MASS) Group. Final follow-up of the Multicentre Aneurysm Screening Study (MASS) randomized trial of abdominal aortic aneurysm screening. Br J Surg 2012;99(12):1649-56. doi: 10.1002/bjs.8897.

6. Jacomelli J, Summers L, Stevenson A, Lees T, Earnshaw JJ. Impact of the first 5 years of a national abdominal aortic aneurysm screening programme. Br J Surg 2016;103(9):1125-31. doi: 10.1002/bjs.10173.

7. Wanhainen $A A$, Hultgren $R$, Linné $A$, et al. Outcome of the Swedish nationwide abdominal aortic aneurysm screening program. Circulation 2016;134(16):1141-48.

8. McCaul KA, Lawrence-Brown M, Dickinson JA, Norman PE. Long-term outcomes of the Western Australian trial of screening for abdominal aortic aneurysms: Secondary analysis of a randomized clinical trial. JAMA Intern Med 2016;176(12):176167. doi: 10.1001/jamainternmed.2016.6633.

9. Lindholt JS, Søgaard R. Population screening and intervention for vascular disease in Danish men (VIVA): A randomised controlled trial. Lancet 2017;390(10109):2256-65. doi: 10.1016/S01406736(17)32250-X.

10. Takagi $H$, Ando T, Umemoto T, ALICE (AllLiterature Investigation of Cardiovascular Evidence) Group. Abdominal aortic aneurysm screening reduces all-cause mortality: Make screening great again. Angiology 2018;69(3):205-11. doi: 10.1177/0003319717693107.

11. Zucker EJ, Misono AS, Prabhakar AM. Abdominal aortic aneurysm screening practices: Impact of the 2014 US Preventive Services Task Force recommendations. J Am Coll Radiol 2017;14(7):868-74.

12. Chaer RA, Vasoncelos R, Marone LK, et al. Synchronous and metachronous thoracic aneurysms in patients with abdominal aortic aneurysms. J Vasc Surg 2012;56(5):1261-65. doi: 10.1016/j.jvs.2012.04.056. 
13. Coles-Black J, Birch B, van der Werff L, Chuen J. An easily reproducible $3 \mathrm{D}$ printed AAA model using open source digital tools. Australian \& New Zealand Society for Vascular Surgery conference 2016; August; Sydney, NSW.

14. McGloughlin TM, Doyle BJ. New approaches to abdominal aortic aneurysm rupture risk assessment: Engineering insights with clinical gain. Arterioscler Thromb Vasc Biol 2010;30(9):1687-94. doi: 10.1161/ ATVBAHA.110.204529.

15. Wanhainen AA, Mani K, Golledge J. Surrogate markers of abdominal aortic aneurysm progression. Arterioscler Thromb Vasc Biol 2016;36(2):236-44. doi: 10.1161/ ATVBAHA.115.306538.

16. United Kingdom Small Aneurysm Trial Participants, Powell JT, Brady AR, et al. Long-term outcomes of immediate repair compared with surveillance of small abdominal aortic aneurysms. N Engl J Med 2002;346(19):1445-52.

17. Moll FL, Powell JT, Fraedrich G, et al. Management of abdominal aortic aneurysms clinical practice guidelines of the European society for vascular surgery. Eur J Vasc Endovasc Surg 2011;41 Suppl 1:S1-58. doi: 10.1016/j.ejvs.2010.09.011.

18. Brady AR, Thompson SG, Fowkes FGR Greenhalgh RM, Powell JT, UK Small Aneurysm Trial Participants. Abdominal aortic aneurysm expansion: Risk factors and time intervals for surveillance. Circulation 2004;110(1):16-21.
19. Chaikof EL, Dalman RL, Eskandari MK, et al. The Society for Vascular Surgery practice guidelines on the care of patients with an abdominal aortic aneurysm. J Vasc Surg 2018;67(1):2-77.e2. doi: 10.1016/j.jvs.2017.10.044

20. RESCAN Collaborators, Bown MJ, Sweeting MJ, Brown LC, Powell JT, Thompson SG. Surveillance intervals for small abdominal aortic aneurysms: A meta-analysis. JAMA 2013;309(8):806-13. doi: 10.1001/jama.2013.950.

21. Van Damme $H$, Deprez $M$, Creemers E, Limet R. Intrinsic structural failure of polyester (Dacron) vascular grafts. A general review. Acta Chir Belg 2005;105(3):249-55.

22. Hallett JW Jr, Marshall DM, Petterson TM, et al. Graft-related complications after abdominal aortic aneurysm repair: Reassurance from a 36-year population-based experience. J Vasc Surg 1997:25(2):277-86.

23. Johnstone JK, Oderich GS. Mechanisms of EVAR failure and new surveillance strategies. Endovascular Today 2014;(2):64-7.

24. Patel R, Sweeting MJ, Powell JT, Greenhalgh RM, EVAR trial investigators. Endovascular versus open repair of abdominal aortic aneurysm in 15-years' follow-up of the UK endovascular aneurysm repair trial 1 (EVAR trial 1): A randomised controlled trial. Lancet 2016;388(10058):2366-74. doi: 10.1016/ S0140-6736(16)31135-7.
25. Sternbergh WC III, Greenberg RK, Chuter TAM, Tonnessen $\mathrm{BH}$, Zenith Investigators. Redefining postoperative surveillance after endovascular aneurysm repair: Recommendations based on 5-year follow-up in the US Zenith multicenter trial. J Vasc Surg 2008;48(2):278-85. doi: 10.1016/j. jvs.2008.02.075.

26. Troutman DA, Chaudry M, Dougherty MJ, Calligaro KD. Endovascular aortic aneurysm repair surveillance may not be necessary for the first 3 years after an initially normal duplex postoperative study. J Vasc Surg 2014;60(3):558-62. doi: 10.1016/j.jvs.2014.03.278.

27. Partovi S, Kaspar M, Aschwanden M, et al. Contrast-enhanced ultrasound after endovascular aortic repair-current status and future perspectives. Cardiovasc Diagn Ther AME 2015:5(6):454-63. doi: 10.3978/j.issn.22233652.2015.09.04

correspondence ajgp@racgp.org.au 